\title{
On coupled common fixed points for mixed weakly monotone maps in partially ordered $S$-metric spaces
}

Nguyen Van Dung*

\section{"Correspondence:}

nvdung@dthu.edu.vn;

nguyendungtc@gmail.com

Department of Mathematics, Dong

Thap University, Cao Lanh, Dong

Thap, Vietnam

\begin{abstract}
In this paper, we use the notion of a mixed weakly monotone pair of maps of Gordji et al. (Fixed Point Theory Appl. 2012:95, 2012) to state a coupled common fixed point theorem for maps on partially ordered S-metric spaces. This result generalizes the main results of Gordji et al. (Fixed Point Theory Appl. 2012:95, 2012), Bhaskar, Lakshmikantham (Nonlinear Anal. 65(7):1379-1393, 2006), Kadelburg et al. (Comput. Math. Appl. 59:3148-3159, 2010) into the structure of S-metric spaces.
\end{abstract}

\section{Introduction and preliminaries}

There are many generalized metric spaces such as 2-metric spaces [1], G-metric spaces [2], $D^{\prime \prime}$-metric spaces [3], partial metric spaces [4] and cone metric spaces [5]. These notions have been investigated by many authors and various versions of fixed point theorems have been stated in [6-23] recently. In [24], Sedghi, Shobe and Aliouche have introduced the notion of an $S$-metric space and proved that this notion is a generalization of a $G$-metric space and a $D "$-metric space. Also, they have proved some properties of $S$-metric spaces and some fixed point theorems for a self-map on an $S$-metric space. An interesting work that naturally rises is to transport certain results in metric spaces and known generalized metric spaces to $S$-metric spaces. In this way, some results have been obtained in [24-26].

In [27], Gordji et al. have introduced the concept of a mixed weakly monotone pair of maps and proved some coupled common fixed point theorems for a contractive-type maps with the mixed weakly monotone property in partially ordered metric spaces. These results give rise to stating coupled common fixed point theorems for maps with the mixed weakly monotone property in partially ordered $S$-metric spaces.

In this paper, we use the notion of a mixed weakly monotone pair of maps to state a coupled common fixed point theorem for maps on partially ordered $S$-metric spaces. This result generalizes the main results of $[6,27,28]$ into the structure of $S$-metric spaces.

First we recall some notions, lemmas and examples which will be useful later.

Definition 1.1 [24, Definition 2.1] Let $X$ be a nonempty set. An $S$-metric on $X$ is a function $S: X^{3} \longrightarrow[0, \infty)$ that satisfies the following conditions for all $x, y, z, a \in X:$

1. $S(x, y, z)=0$ if and only if $x=y=z$.

2. $S(x, y, z) \leq S(x, x, a)+S(y, y, a)+S(z, z, a)$.

The pair $(X, S)$ is called an $S$-metric space. 
The following is an intuitive geometric example for $S$-metric spaces.

Example 1.2 [24, Example 2.4] Let $X=\mathbb{R}^{2}$ and $d$ be an ordinary metric on $X$. Put

$$
S(x, y, z)=d(x, y)+d(x, z)+d(y, z)
$$

for all $x, y, z \in \mathbb{R}^{2}$, that is, $S$ is the perimeter of the triangle given by $x, y, z$. Then $S$ is an $S$-metric on $X$.

Lemma 1.3 [24, Lemma 2.5] Let $(X, S)$ be an S-metric space. Then $S(x, x, y)=S(y, y, x)$ for all $x, y \in X$.

The following lemma is a direct consequence of Definition 1.1 and Lemma 1.3.

Lemma 1.4 [25, Lemma 1.6] Let $(X, S)$ be an S-metric space. Then

$$
S(x, x, z) \leq 2 S(x, x, y)+S(y, y, z)
$$

and

$$
S(x, x, z) \leq 2 S(x, x, y)+S(z, z, y)
$$

for all $x, y, z \in X$.

Definition 1.5 [24, Definition 2.8] Let $(X, S)$ be an $S$-metric space.

1. A sequence $\left\{x_{n}\right\} \subset X$ is said to converge to $x \in X$ if $S\left(x_{n}, x_{n}, x\right) \rightarrow 0$ as $n \rightarrow \infty$. That is, for each $\varepsilon>0$, there exists $n_{0} \in \mathbb{N}$ such that for all $n \geq n_{0}$ we have $S\left(x_{n}, x_{n}, x\right)<\varepsilon$. We write $x_{n} \rightarrow x$ for brevity.

2. A sequence $\left\{x_{n}\right\} \subset X$ is called a Cauchy sequence if $S\left(x_{n}, x_{n}, x_{m}\right) \rightarrow 0$ as $n, m \rightarrow \infty$. That is, for each $\varepsilon>0$, there exists $n_{0} \in \mathbb{N}$ such that for all $n, m \geq n_{0}$ we have $S\left(x_{n}, x_{n}, x_{m}\right)<\varepsilon$.

3. The $S$-metric space $(X, S)$ is said to be complete if every Cauchy sequence is a convergent sequence.

From [24, Examples on p.260] we have the following.

\section{Example 1.6}

1. Let $\mathbb{R}$ be a real line. Then

$$
S(x, y, z)=|x-z|+|y-z|
$$

for all $x, y, z \in \mathbb{R}$ is an $S$-metric on $\mathbb{R}$. This $S$-metric is called the usual $S$-metric on $\mathbb{R}$. Furthermore, the usual $S$-metric space $\mathbb{R}$ is complete.

2. Let $Y$ be a nonempty subset of $\mathbb{R}$. Then

$$
S(x, y, z)=|x-z|+|y-z|
$$

for all $x, y, z \in Y$ is an $S$-metric on $Y$. Furthermore, if $Y$ is a closed subset of the usual metric space $\mathbb{R}$, then the $S$-metric space $Y$ is complete. 
Lemma 1.7 [24, Lemma 2.12] Let $(X, S)$ be an S-metric space. If $x_{n} \rightarrow x$ and $y_{n} \rightarrow y$, then $S\left(x_{n}, x_{n}, y_{n}\right) \rightarrow S(x, x, y)$.

Definition 1.8 [24] Let $(X, S)$ be an S-metric space. For $r>0$ and $x \in X$, we define the open ball $B_{S}(x, r)$ and the closed ball $B_{S}[x, r]$ with center $x$ and radius $r$ as follows:

$$
\begin{aligned}
& B_{S}(x, r)=\{y \in X: S(y, y, x)<r\}, \\
& B_{S}[x, r]=\{y \in X: S(y, y, x) \leq r\} .
\end{aligned}
$$

The topology induced by the S-metric or the S-metric topology is the topology generated by the base of all open balls in $X$.

Lemma 1.9 Let $\left\{x_{n}\right\}$ be a sequence in $X$. Then $x_{n} \rightarrow x$ in the S-metric space $(X, S)$ if and only if $x_{n} \rightarrow x$ in the $S$-metric topological space $X$.

Proof It is a direct consequence of Definition 1.5(1) and Definition 1.8.

The following lemma shows that every metric space is an $S$-metric space.

Lemma 1.10 Let $(X, d)$ be a metric space. Then we have

1. $S_{d}(x, y, z)=d(x, z)+d(y, z)$ for all $x, y, z \in X$ is an $S$-metric on $X$.

2. $x_{n} \rightarrow x$ in $(X, d)$ if and only if $x_{n} \rightarrow x$ in $\left(X, S_{d}\right)$.

3. $\left\{x_{n}\right\}$ is Cauchy in $(X, d)$ if and only if $\left\{x_{n}\right\}$ is Cauchy in $\left(X, S_{d}\right)$.

4. $(X, d)$ is complete if and only if $\left(X, S_{d}\right)$ is complete.

\section{Proof}

1. See [24, Example (3), p.260].

2. $x_{n} \rightarrow x$ in $(X, d)$ if and only if $d\left(x_{n}, x\right) \rightarrow 0$, if and only if

$$
S_{d}\left(x_{n}, x_{n}, x\right)=2 d\left(x_{n}, x\right) \rightarrow 0,
$$

that is, $x_{n} \rightarrow x$ in $\left(X, S_{d}\right)$.

3. $\left\{x_{n}\right\}$ is Cauchy in $(X, d)$ if and only if $d\left(x_{n}, x_{m}\right) \rightarrow 0$ as $n, m \rightarrow \infty$, if and only if

$$
S_{d}\left(x_{n}, x_{n}, x_{m}\right)=2 d\left(x_{n}, x_{m}\right) \rightarrow 0
$$

as $n, m \rightarrow \infty$, that is, $\left\{x_{n}\right\}$ is Cauchy in $\left(X, S_{d}\right)$.

4. It is a direct consequence of (2) and (3).

The following example proves that the inverse implication of Lemma 1.10 does not hold.

Example 1.11 Let $X=\mathbb{R}$ and $S(x, y, z)=|y+z-2 x|+|y-z|$ for all $x, y, z \in X$. By [24, Example (1), p.260], $(X, S)$ is an $S$-metric space. We will prove that there does not exist any metric $d$ such that $S(x, y, z)=d(x, z)+d(y, z)$ for all $x, y, z \in X$. Indeed, suppose to the contrary that there exists a metric $d$ with $S(x, y, z)=d(x, z)+d(y, z)$ for all $x, y, z \in X$. Then $d(x, z)=\frac{1}{2} S(x, x, z)=|x-z|$ and $d(x, y)=S(x, y, y)=2|x-y|$ for all $x, y, z \in X$. It is a contradiction. 
Lemma 1.12 [27, p.7] Let $(X, d)$ be a metric space. Then $X \times X$ is a metric space with the metric $D_{d}$ given by

$$
D_{d}((x, y),(u, v))=d(x, u)+d(y, v)
$$

for all $x, y, u, v \in X$.

Lemma 1.13 Let $(X, S)$ be an $S$-metric space. Then $X \times X$ is an $S$-metric space with the $S$-metric $D$ given by

$$
D((x, y),(u, v),(z, w))=S(x, u, z)+S(y, v, w)
$$

for all $x, y, u, v, z, w \in X$.

Proof For all $x, y, u, v, z, w \in X$, we have $D((x, y),(u, v),(z, w)) \in[0, \infty)$ and

$$
D((x, y),(u, v),(z, w))=0 \quad \text { if and only if } \quad S(x, u, z)+S(y, v, w)=0
$$

if and only if $x=u=z, y=v=w$, that is, $(x, y)=(u, v)=(z, w)$; and

$$
\begin{aligned}
D & ((x, y),(u, v),(z, w)) \\
& =S(x, u, z)+S(y, v, w) \\
& \leq S(x, x, a)+S(u, u, a)+S(z, z, a)+S(y, y, b)+S(v, v, b)+S(w, w, b) \\
& =D((x, y),(x, y),(a, b))+D((u, v),(u, v),(a, b))+D((z, w),(z, w),(a, b)) .
\end{aligned}
$$

By the above, $D$ is an $S$-metric on $X \times X$.

Remark 1.14 Let $(X, d)$ be a metric space. By using Lemma 1.13 with $S=S_{d}$, we get

$$
D((x, y),(x, y),(u, v))=S_{d}(x, x, u)+S_{d}(y, y, v)=2(d(x, u)+d(y, v))=2 D_{d}((x, y),(u, v))
$$

for all $x, y, u, v \in X$.

Lemma 1.15 [17, p.4] Let $(X, \preceq)$ be a partially ordered set. Then $X \times X$ is a partially ordered set with the partial order $\preceq$ defined by

$$
(x, y) \preceq(u, v) \quad \text { if and only if } \quad x \preceq u, \quad v \preceq y .
$$

Remark 1.16 Let $X$ be a subset of $\mathbb{R}$ with the usual order. For each $\left(x_{1}, x_{2}\right),\left(y_{1}, y_{2}\right) \in X \times$ $X$, put $z_{1}=\max \left\{x_{1}, y_{1}\right\}$ and $z_{2}=\min \left\{x_{2}, y_{2}\right\}$, then $\left(x_{1}, x_{2}\right) \preceq\left(z_{1}, z_{2}\right)$ and $\left(y_{1}, y_{2}\right) \preceq\left(z_{1}, z_{2}\right)$. Therefore, for each $\left(x_{1}, x_{2}\right),\left(y_{1}, y_{2}\right) \in X \times X$, there exists $\left(z_{1}, z_{2}\right) \in X \times X$ that is comparable to $\left(x_{1}, x_{2}\right)$ and $\left(y_{1}, y_{2}\right)$.

Definition 1.17 [27, Definition 1.5] Let $(X, \preceq)$ be a partially ordered set and $f, g: X \times X \rightarrow$ $X$ be two maps. We say that a pair $(f, g)$ has the mixed weakly monotone property on $X$ if, 
for all $x, y \in X$, we have

$$
x \preceq f(x, y), f(y, x) \preceq y \quad \text { implies } f(x, y) \preceq g(f(x, y), f(y, x)), g(f(y, x), f(x, y)) \preceq f(y, x)
$$

and

$$
x \preceq g(x, y), g(y, x) \preceq y \quad \text { implies } \quad g(x, y) \preceq f(g(x, y), g(y, x)), f(g(y, x), g(x, y)) \preceq g(y, x) .
$$

Example 1.18 [27, Example 1.6] Let $f, g: \mathbb{R} \times \mathbb{R} \rightarrow \mathbb{R}$ be two functions given by

$$
f(x, y)=x-2 y, \quad g(x, y)=x-y .
$$

Then the pair $(f, g)$ has the mixed weakly monotone property.

Example 1.19 [27, Example 1.7] Let $f, g: \mathbb{R} \times \mathbb{R} \longrightarrow \mathbb{R}$ be two functions given by

$$
f(x, y)=x-y+1, \quad g(x, y)=2 x-3 y .
$$

Then $f$ and $g$ have the mixed monotone property but the pair $(f, g)$ does not have the mixed weakly monotone property.

Remark 1.20 [27, Remark 2.5] Let $(X, \preceq)$ be a partially ordered set; $f: X \times X \rightarrow X$ be a map with the mixed monotone property on $X$. Then for all $n \in \mathbb{N}$, the pair $\left(f^{n}, f^{n}\right)$ has the mixed weakly monotone property on $X$.

\section{Main results}

Theorem 2.1 Let $(X, \preceq, S)$ be a partially ordered S-metric space; $f, g: X \times X \rightarrow X$ be two maps such that

1. $X$ is complete;

2. The pair $(f, g)$ has the mixed weakly monotone property on $X$; $x_{0} \preceq f\left(x_{0}, y_{0}\right), f\left(y_{0}, x_{0}\right) \preceq y_{0}$ or $x_{0} \preceq g\left(x_{0}, y_{0}\right), g\left(y_{0}, x_{0}\right) \preceq y_{0}$ for some $x_{0}, y_{0} \in X$;

3. There exist $p, q, r, s \geq 0$ satisfying $p+q+r+2 s<1$ and

$$
\begin{aligned}
& S(f(x, y), f(x, y), g(u, v)) \\
& \leq \frac{p}{2} D((x, y),(x, y),(u, v))+\frac{q}{2} D((x, y),(x, y),(f(x, y), f(y, x))) \\
&+\frac{r}{2} D((u, v),(u, v),(g(u, v), g(v, u)))+\frac{s}{2} D((x, y),(x, y),(g(u, v), g(v, u))) \\
&+\frac{s}{2} D((u, v),(u, v),(f(x, y), f(y, x)))
\end{aligned}
$$

for all $x, y, u, v \in X$ with $x \preceq u$ and $y \geq v$ where $D$ is defined as in Lemma 1.13;

4. $f$ or $g$ is continuous or $X$ has the following property:

(a) If $\left\{x_{n}\right\}$ is an increasing sequence with $x_{n} \rightarrow x$, then $x_{n} \preceq x$ for all $n \in \mathbb{N}$;

(b) If $\left\{x_{n}\right\}$ is an decreasing sequence with $x_{n} \rightarrow x$, then $x \preceq x_{n}$ for all $n \in \mathbb{N}$.

Then $f$ and $g$ have a coupled common fixed point in $X$. 
Proof First we note that the roles of $f$ and $g$ can be interchanged in the assumptions. We need only prove the case $x_{0} \preceq f\left(x_{0}, y_{0}\right)$ and $f\left(y_{0}, x_{0}\right) \preceq y_{0}$, the case $x_{0} \preceq g\left(x_{0}, y_{0}\right)$ and $g\left(y_{0}, x_{0}\right) \preceq y_{0}$ is proved similarly by interchanging the roles of $f$ and $g$.

Step 1 . We construct two Cauchy sequences in $X$.

Put $x_{1}=f\left(x_{0}, y_{0}\right), y_{1}=f\left(y_{0}, x_{0}\right)$. Since $(f, g)$ has the mixed weakly monotone property, we have

$$
x_{1}=f\left(x_{0}, y_{0}\right) \preceq g\left(f\left(x_{0}, y_{0}\right), f\left(y_{0}, x_{0}\right)\right)=g\left(x_{1}, y_{1}\right)
$$

and

$$
y_{1}=f\left(y_{0}, x_{0}\right) \succeq g\left(f\left(y_{0}, x_{0}\right), f\left(x_{0}, y_{0}\right)\right)=g\left(y_{1}, x_{1}\right) .
$$

Put $x_{2}=g\left(x_{1}, y_{1}\right), y_{2}=g\left(y_{1}, x_{1}\right)$. Then we have

$$
x_{2}=g\left(x_{1}, y_{1}\right) \preceq f\left(g\left(x_{1}, y_{1}\right), g\left(y_{1}, x_{1}\right)\right)=f\left(x_{2}, y_{2}\right)
$$

and

$$
y_{2}=g\left(y_{1}, x_{1}\right) \succeq f\left(g\left(y_{1}, x_{1}\right), g\left(x_{1}, y_{1}\right)\right)=f\left(y_{2}, x_{2}\right) .
$$

Continuously, for all $n \in \mathbb{N}$, we put

$$
\begin{aligned}
& x_{2 n+1}=f\left(x_{2 n}, y_{2 n}\right), \quad y_{2 n+1}=f\left(y_{2 n}, x_{2 n}\right), \\
& x_{2 n+2}=g\left(x_{2 n+1}, y_{2 n+1}\right), \quad y_{2 n+2}=g\left(y_{2 n+1}, x_{2 n+1}\right)
\end{aligned}
$$

that satisfy

$$
x_{0} \preceq x_{1} \preceq \cdots \preceq x_{n} \preceq \cdots \quad \text { and } \quad y_{0} \succeq y_{1} \succeq \cdots \succeq y_{n} \succeq \cdots .
$$

We will prove that $\left\{x_{n}\right\}$ and $\left\{y_{n}\right\}$ are two Cauchy sequences. For all $n \in \mathbb{N}$, it follows from (2.1) that

$$
\begin{aligned}
& S\left(x_{2 n+1}, x_{2 n+1}, x_{2 n+2}\right) \\
&=S\left(f\left(x_{2 n}, y_{2 n}\right), f\left(x_{2 n}, y_{2 n}\right), g\left(x_{2 n+1}, y_{2 n+1}\right)\right) \\
& \leq \frac{p}{2} D\left(\left(x_{2 n}, y_{2 n}\right),\left(x_{2 n}, y_{2 n}\right),\left(x_{2 n+1}, y_{2 n+1}\right)\right) \\
&+\frac{q}{2} D\left(\left(x_{2 n}, y_{2 n}\right),\left(x_{2 n}, y_{2 n}\right),\left(f\left(x_{2 n}, y_{2 n}\right), f\left(y_{2 n}, x_{2 n}\right)\right)\right) \\
&+\frac{r}{2} D\left(\left(x_{2 n+1}, y_{2 n+1}\right),\left(x_{2 n+1}, y_{2 n+1}\right),\left(g\left(x_{2 n+1}, y_{2 n+1}\right), g\left(y_{2 n+1}, x_{2 n+1}\right)\right)\right) \\
&+\frac{s}{2} D\left(\left(x_{2 n}, y_{2 n}\right),\left(x_{2 n}, y_{2 n}\right),\left(g\left(x_{2 n+1}, y_{2 n+1}\right), g\left(y_{2 n+1}, x_{2 n+1}\right)\right)\right) \\
&+\frac{s}{2} D\left(\left(x_{2 n+1}, y_{2 n+1}\right),\left(x_{2 n+1}, y_{2 n+1}\right),\left(f\left(x_{2 n}, y_{2 n}\right), f\left(y_{2 n}, x_{2 n}\right)\right)\right) .
\end{aligned}
$$


By using (2.2) we get

$$
\begin{aligned}
S\left(x_{2 n+1}, x_{2 n+1}, x_{2 n+2}\right) \leq & \frac{p}{2} D\left(\left(x_{2 n}, y_{2 n}\right),\left(x_{2 n}, y_{2 n}\right),\left(x_{2 n+1}, y_{2 n+1}\right)\right) \\
& +\frac{q}{2} D\left(\left(x_{2 n}, y_{2 n}\right),\left(x_{2 n}, y_{2 n}\right),\left(x_{2 n+1}, y_{2 n+1}\right)\right) \\
& +\frac{r}{2} D\left(\left(x_{2 n+1}, y_{2 n+1}\right),\left(x_{2 n+1}, y_{2 n+1}\right),\left(x_{2 n+2}, y_{2 n+2}\right)\right) \\
& +\frac{s}{2} D\left(\left(x_{2 n}, y_{2 n}\right),\left(x_{2 n}, y_{2 n}\right),\left(x_{2 n+2}, y_{2 n+2}\right)\right) \\
& +\frac{s}{2} D\left(\left(x_{2 n+1}, y_{2 n+1}\right),\left(x_{2 n+1}, y_{2 n+1}\right),\left(x_{2 n+1}, y_{2 n+1}\right)\right) \\
= & \frac{p+q}{2} D\left(\left(x_{2 n}, y_{2 n}\right),\left(x_{2 n}, y_{2 n}\right),\left(x_{2 n+1}, y_{2 n+1}\right)\right) \\
& +\frac{r}{2} D\left(\left(x_{2 n+1}, y_{2 n+1}\right),\left(x_{2 n+1}, y_{2 n+1}\right),\left(x_{2 n+2}, y_{2 n+2}\right)\right) \\
& +\frac{s}{2} D\left(\left(x_{2 n}, y_{2 n}\right),\left(x_{2 n}, y_{2 n}\right),\left(x_{2 n+2}, y_{2 n+2}\right)\right) \\
\leq & \frac{p+q+s}{2} D\left(\left(x_{2 n}, y_{2 n}\right),\left(x_{2 n}, y_{2 n}\right),\left(x_{2 n+1}, y_{2 n+1}\right)\right) \\
& +\frac{r+s}{2} D\left(\left(x_{2 n+1}, y_{2 n+1}\right),\left(x_{2 n+1}, y_{2 n+1}\right),\left(x_{2 n+2}, y_{2 n+2}\right)\right) .
\end{aligned}
$$

That is,

$$
\begin{aligned}
& S\left(x_{2 n+1}, x_{2 n+1}, x_{2 n+2}\right) \\
& \quad \leq \frac{p+q+s}{2}\left(S\left(x_{2 n}, x_{2 n}, x_{2 n+1}\right)+S\left(y_{2 n}, y_{2 n}, y_{2 n+1}\right)\right) \\
& \quad+\frac{r+s}{2}\left(S\left(x_{2 n+1}, x_{2 n+1}, x_{2 n+2}\right)+S\left(y_{2 n+1}, y_{2 n+1}, y_{2 n+2}\right)\right) .
\end{aligned}
$$

Analogously to (2.4), we have

$$
\begin{aligned}
& S\left(y_{2 n+1}, y_{2 n+1}, y_{2 n+2}\right) \\
& \quad \leq \frac{p+q+s}{2}\left(S\left(y_{2 n}, y_{2 n}, y_{2 n+1}\right)+S\left(x_{2 n}, x_{2 n}, x_{2 n+1}\right)\right) \\
& \quad+\frac{r+s}{2}\left(S\left(y_{2 n+1}, y_{2 n+1}, y_{2 n+2}\right)+S\left(x_{2 n+1}, x_{2 n+1}, x_{2 n+2}\right)\right) .
\end{aligned}
$$

It follows from (2.4) and (2.5) that

$$
\begin{aligned}
& S\left(x_{2 n+1}, x_{2 n+1}, x_{2 n+2}\right)+S\left(y_{2 n+1}, y_{2 n+1}, y_{2 n+2}\right) \\
& \quad \leq \frac{p+q+s}{1-(r+s)}\left(S\left(x_{2 n}, x_{2 n}, x_{2 n+1}\right)+S\left(y_{2 n}, y_{2 n}, y_{2 n+1}\right)\right) .
\end{aligned}
$$

For all $n \in \mathbb{N}$, by interchanging the roles of $f$ and $g$ and using (2.1) again, we have

$$
\begin{aligned}
& S\left(x_{2 n+2}, x_{2 n+2}, x_{2 n+3}\right) \\
& \quad=S\left(g\left(x_{2 n+1}, y_{2 n+1}\right), g\left(x_{2 n+1}, y_{2 n+1}\right), f\left(x_{2 n+2}, y_{2 n+2}\right)\right) \\
& \quad \leq \frac{p}{2} D\left(\left(x_{2 n+1}, y_{2 n+1}\right),\left(x_{2 n+1}, y_{2 n+1}\right),\left(x_{2 n+2}, y_{2 n+2}\right)\right)
\end{aligned}
$$




$$
\begin{aligned}
& +\frac{q}{2} D\left(\left(x_{2 n+1}, y_{2 n+1}\right),\left(x_{2 n+1}, y_{2 n+1}\right),\left(g\left(x_{2 n+1}, y_{2 n+1}\right), g\left(y_{2 n+1}, x_{2 n+1}\right)\right)\right) \\
& +\frac{r}{2} D\left(\left(x_{2 n+2}, y_{2 n+2}\right),\left(x_{2 n+2}, y_{2 n+2}\right),\left(f\left(x_{2 n+2}, y_{2 n+2}\right), f\left(y_{2 n+2}, x_{2 n+2}\right)\right)\right) \\
& +\frac{s}{2} D\left(\left(x_{2 n+1}, y_{2 n+1}\right),\left(x_{2 n+1}, y_{2 n+1}\right),\left(f\left(x_{2 n+2}, y_{2 n+2}\right), f\left(y_{2 n+2}, x_{2 n+2}\right)\right)\right) \\
& +\frac{s}{2} D\left(\left(x_{2 n+2}, y_{2 n+2}\right),\left(x_{2 n+2}, y_{2 n+2}\right),\left(g\left(x_{2 n+1}, y_{2 n+1}\right), g\left(y_{2 n+1}, x_{2 n+1}\right)\right)\right) .
\end{aligned}
$$

By using (2.2) we get

$$
\begin{aligned}
S\left(x_{2 n+2}, x_{2 n+2}, x_{2 n+3}\right) \leq & \frac{p}{2} D\left(\left(x_{2 n+1}, y_{2 n+1}\right),\left(x_{2 n+1}, y_{2 n+1}\right),\left(x_{2 n+2}, y_{2 n+2}\right)\right) \\
& +\frac{q}{2} D\left(\left(x_{2 n+1}, y_{2 n+1}\right),\left(x_{2 n+1}, y_{2 n+1}\right),\left(x_{2 n+2}, y_{2 n+2}\right)\right) \\
& +\frac{r}{2} D\left(\left(x_{2 n+2}, y_{2 n+2}\right),\left(x_{2 n+2}, y_{2 n+2}\right),\left(x_{2 n+3}, y_{2 n+3}\right)\right) \\
& +\frac{s}{2} D\left(\left(x_{2 n+1}, y_{2 n+1}\right),\left(x_{2 n+1}, y_{2 n+1}\right),\left(x_{2 n+3}, y_{2 n+3}\right)\right) \\
& +\frac{s}{2} D\left(\left(x_{2 n+2}, y_{2 n+2}\right),\left(x_{2 n+2}, y_{2 n+2}\right),\left(x_{2 n+2}, y_{2 n+2}\right)\right) \\
= & \frac{p+q}{2} D\left(\left(x_{2 n+1}, y_{2 n+1}\right),\left(x_{2 n+1}, y_{2 n+1}\right),\left(x_{2 n+2}, y_{2 n+2}\right)\right) \\
& +\frac{r}{2} D\left(\left(x_{2 n+2}, y_{2 n+2}\right),\left(x_{2 n+2}, y_{2 n+2}\right),\left(x_{2 n+3}, y_{2 n+3}\right)\right) \\
& +\frac{s}{2} D\left(\left(x_{2 n+1}, y_{2 n+1}\right),\left(x_{2 n+1}, y_{2 n+1}\right),\left(x_{2 n+3}, y_{2 n+3}\right)\right) \\
\leq & \frac{p+q+s}{2} D\left(\left(x_{2 n+1}, y_{2 n+1}\right),\left(x_{2 n+1}, y_{2 n+1}\right),\left(x_{2 n+2}, y_{2 n+2}\right)\right) \\
& +\frac{r+s}{2} D\left(\left(x_{2 n+2}, y_{2 n+2}\right),\left(x_{2 n+2}, y_{2 n+2}\right),\left(x_{2 n+3}, y_{2 n+3}\right)\right) .
\end{aligned}
$$

That is,

$$
\begin{aligned}
& S\left(x_{2 n+2}, x_{2 n+2}, x_{2 n+3}\right) \\
& \leq \frac{p+q+S}{2}\left(S\left(x_{2 n+1}, x_{2 n+1}, x_{2 n+2}\right)+S\left(y_{2 n+1}, y_{2 n+1}, y_{2 n+2}\right)\right) \\
& \quad+\frac{r+s}{2}\left(S\left(x_{2 n+2}, x_{2 n+2}, x_{2 n+3}\right)+S\left(y_{2 n+2}, y_{2 n+2}, y_{2 n+3}\right)\right) .
\end{aligned}
$$

Analogously to (2.7), we have

$$
\begin{aligned}
& S\left(y_{2 n+2}, y_{2 n+2}, y_{2 n+3}\right) \\
& \leq \frac{p+q+s}{2}\left(S\left(y_{2 n+1}, y_{2 n+1}, y_{2 n+2}\right)+S\left(x_{2 n+1}, x_{2 n+1}, x_{2 n+2}\right)\right) \\
& \quad+\frac{r+s}{2}\left(S\left(y_{2 n+2}, y_{2 n+2}, y_{2 n+3}\right)+S\left(x_{2 n+2}, x_{2 n+2}, x_{2 n+3}\right)\right) .
\end{aligned}
$$

It follows from (2.7) and (2.8) that

$$
\begin{aligned}
& S\left(x_{2 n+2}, x_{2 n+2}, x_{2 n+3}\right)+S\left(y_{2 n+2}, y_{2 n+2}, y_{2 n+3}\right) \\
& \quad \leq \frac{p+q+s}{1-(r+s)}\left(S\left(x_{2 n+1}, x_{2 n+1}, x_{2 n+2}\right)+S\left(y_{2 n+1}, y_{2 n+1}, y_{2 n+2}\right)\right) .
\end{aligned}
$$


For all $n \in \mathbb{N}$, (2.6) and (2.9) combine to give

$$
\begin{aligned}
& S\left(x_{2 n+2}, x_{2 n+2}, x_{2 n+3}\right)+S\left(y_{2 n+2}, y_{2 n+2}, y_{2 n+3}\right) \\
& \quad \leq \frac{p+q+s}{1-(r+s)}\left(S\left(x_{2 n+1}, x_{2 n+1}, x_{2 n+2}\right)+S\left(y_{2 n+1}, y_{2 n+1}, y_{2 n+2}\right)\right) \\
& \quad \leq\left(\frac{p+q+s}{1-(r+s)}\right)^{2}\left(S\left(x_{2 n}, x_{2 n}, x_{2 n+1}\right)+S\left(y_{2 n}, y_{2 n}, y_{2 n+1}\right)\right) .
\end{aligned}
$$

Now we have

$$
\begin{aligned}
& S\left(x_{2 n+1}, x_{2 n+1}, x_{2 n+2}\right)+S\left(y_{2 n+1}, y_{2 n+1}, y_{2 n+2}\right) \\
& \quad \leq \frac{p+q+s}{1-(r+s)}\left(S\left(x_{2 n}, x_{2 n}, x_{2 n+1}\right)+S\left(y_{2 n}, y_{2 n}, y_{2 n+1}\right)\right) \\
& \quad \leq\left(\frac{p+q+s}{1-(r+s)}\right)^{3}\left(S\left(x_{2 n-2}, x_{2 n-2}, x_{2 n-1}\right)+S\left(y_{2 n-2}, y_{2 n-2}, y_{2 n-1}\right)\right) \\
& \quad \ldots \\
& \quad \leq\left(\frac{p+q+s}{1-(r+s)}\right)^{2 n+1}\left(S\left(x_{0}, x_{0}, x_{1}\right)+S\left(y_{0}, y_{0}, y_{1}\right)\right)
\end{aligned}
$$

and

$$
\begin{aligned}
& S\left(x_{2 n+2}, x_{2 n+2}, x_{2 n+3}\right)+S\left(y_{2 n+2}, y_{2 n+2}, y_{2 n+3}\right) \\
& \quad \leq\left(\frac{p+q+s}{1-(r+s)}\right)^{2}\left(S\left(x_{2 n}, x_{2 n}, x_{2 n+1}\right)+S\left(y_{2 n}, y_{2 n}, y_{2 n+1}\right)\right) \\
& \quad \leq\left(\frac{p+q+s}{1-(r+s)}\right)^{4}\left(S\left(x_{2 n-2}, x_{2 n-2}, x_{2 n-1}\right)+S\left(y_{2 n-2}, y_{2 n-2}, y_{2 n-1}\right)\right) \\
& \quad \cdots \\
& \quad \leq\left(\frac{p+q+s}{1-(r+s)}\right)^{2 n+2}\left(S\left(x_{0}, x_{0}, x_{1}\right)+S\left(y_{0}, y_{0}, y_{1}\right)\right) .
\end{aligned}
$$

For all $n, m \in \mathbb{N}$ with $n \leq m$, by using Lemma 1.4 and (2.11), (2.12), we have

$$
\begin{aligned}
& S\left(x_{2 n+1}, x_{2 n+1}, x_{2 m+1}\right)+S\left(y_{2 n+1}, y_{2 n+1}, y_{2 m+1}\right) \\
& \leq\left(2 S\left(x_{2 n+1}, x_{2 n+1}, x_{2 n+2}\right)+2 S\left(y_{2 n+1}, y_{2 n+1}, y_{2 n+2}\right)\right) \\
&+\left(S\left(x_{2 n+2}, x_{2 n+2}, x_{2 m+1}\right)+S\left(y_{2 n+2}, y_{2 n+2}, y_{2 m+1}\right)\right) \\
& \leq\left(2 S\left(x_{2 n+1}, x_{2 n+1}, x_{2 n+2}\right)+2 S\left(y_{2 n+1}, y_{2 n+1}, y_{2 n+2}\right)\right) \\
&+\left(2 S\left(x_{2 n+2}, x_{2 n+2}, x_{2 n+3}\right)+2 S\left(y_{2 n+2}, y_{2 n+2}, y_{2 n+3}\right)\right) \\
&+\cdots+\left(2 S\left(x_{2 m-1}, x_{2 m-1}, x_{2 m}\right)+2 S\left(y_{2 m-1}, y_{2 m-1}, y_{2 m}\right)\right) \\
&+\left(S\left(x_{2 m}, x_{2 m}, x_{2 m+1}\right)+S\left(y_{2 m}, y_{2 m}, y_{2 m+1}\right)\right) \\
& \leq\left(2 S\left(x_{2 n+1}, x_{2 n+1}, x_{2 n+2}\right)+2 S\left(y_{2 n+1}, y_{2 n+1}, y_{2 n+2}\right)\right) \\
&+\cdots+\left(2 S\left(x_{2 m}, x_{2 m}, x_{2 m+1}\right)+2 S\left(y_{2 m}, y_{2 m}, y_{2 m+1}\right)\right) \\
& \leq {\left[\left(\frac{p+q+s}{1-(r+s)}\right)^{2 n+1}+\cdots+\left(\frac{p+q+s}{1-(r+s)}\right)^{2 m}\right] }
\end{aligned}
$$




$$
\begin{aligned}
& \times\left(S\left(x_{0}, x_{0}, x_{1}\right)+S\left(y_{0}, y_{0}, y_{1}\right)\right) \\
\leq & \frac{\left(\frac{p+q+s}{1-(r+s)}\right)^{2 n+1}}{1-\frac{p+q+s}{1-(r+s)}}\left(S\left(x_{0}, x_{0}, x_{1}\right)+S\left(y_{0}, y_{0}, y_{1}\right)\right) .
\end{aligned}
$$

Similarly, we have

$$
\begin{aligned}
& S\left(x_{2 n}, x_{2 n}, x_{2 m+1}\right)+S\left(y_{2 n}, y_{2 n}, y_{2 m+1}\right) \\
& \quad \leq\left[\left(\frac{p+q+s}{1-(r+s)}\right)^{2 n}+\cdots+\left(\frac{p+q+s}{1-(r+s)}\right)^{2 m}\right]\left(S\left(x_{0}, x_{0}, x_{1}\right)+S\left(y_{0}, y_{0}, y_{1}\right)\right) \\
& \quad \leq \frac{\left(\frac{p+q+s}{1-(r+s)}\right)^{2 n}}{1-\frac{p+q+s}{1-(r+s)}}\left(S\left(x_{0}, x_{0}, x_{1}\right)+S\left(y_{0}, y_{0}, y_{1}\right)\right)
\end{aligned}
$$

and

$$
\begin{aligned}
& S\left(x_{2 n}, x_{2 n}, x_{2 m}\right)+S\left(y_{2 n}, y_{2 n}, y_{2 m}\right) \\
& \quad \leq\left[\left(\frac{p+q+s}{1-(r+s)}\right)^{2 n}+\cdots+\left(\frac{p+q+s}{1-(r+s)}\right)^{2 m-1}\right]\left(S\left(x_{0}, x_{0}, x_{1}\right)+S\left(y_{0}, y_{0}, y_{1}\right)\right) \\
& \quad \leq \frac{\left(\frac{p+q+s}{1-(r+s)}\right)^{2 n}}{1-\frac{p+q+s}{1-(r+s)}}\left(S\left(x_{0}, x_{0}, x_{1}\right)+S\left(y_{0}, y_{0}, y_{1}\right)\right)
\end{aligned}
$$

and

$$
\begin{aligned}
& S\left(x_{2 n+1}, x_{2 n+1}, x_{2 m}\right)+S\left(y_{2 n+1}, y_{2 n+1}, y_{2 m}\right) \\
& \quad \leq\left[\left(\frac{p+q+s}{1-(r+s)}\right)^{2 n+1}+\cdots+\left(\frac{p+q+s}{1-(r+s)}\right)^{2 m-1}\right]\left(S\left(x_{0}, x_{0}, x_{1}\right)+S\left(y_{0}, y_{0}, y_{1}\right)\right) \\
& \quad \leq \frac{\left(\frac{p+q+s}{1-(r+s)}\right)^{2 n+1}}{1-\frac{p+q+s}{1-(r+s)}}\left(S\left(x_{0}, x_{0}, x_{1}\right)+S\left(y_{0}, y_{0}, y_{1}\right)\right) .
\end{aligned}
$$

Hence, for all $n, m \in \mathbb{N}$ with $n \leq m$, it follows that

$$
S\left(x_{n}, x_{n}, x_{m}\right)+S\left(y_{n}, y_{n}, y_{m}\right) \leq \frac{\left(\frac{p+q+s}{1-(r+s)}\right)^{2 n}}{1-\frac{p+q+s}{1-(r+s)}}\left(S\left(x_{0}, x_{0}, x_{1}\right)+S\left(y_{0}, y_{0}, y_{1}\right)\right) .
$$

Since $0 \leq \frac{p+q+s}{1-(r+s)}<1$, taking the limit as $n, m \rightarrow \infty$, we get

$$
\lim _{n, m \rightarrow \infty}\left(S\left(x_{n}, x_{n}, x_{m}\right)+S\left(y_{n}, y_{n}, y_{m}\right)\right)=0 .
$$

It implies that

$$
\lim _{n, m \rightarrow \infty} S\left(x_{n}, x_{n}, x_{m}\right)=\lim _{n, m \rightarrow \infty} S\left(y_{n}, y_{n}, y_{m}\right)=0 .
$$

Therefore, $\left\{x_{n}\right\}$ and $\left\{y_{n}\right\}$ are two Cauchy sequences in $X$. Since $X$ is complete, there exist $x, y \in X$ such that $x_{n} \rightarrow x$ and $y_{n} \rightarrow y$ in $X$ as $n \rightarrow \infty$.

Step 2. We prove that $(x, y)$ is a coupled common fixed point of $f$ and $g$. We consider the following two cases. 
Case 2.1. $f$ is continuous. We have

$$
x=\lim _{n \rightarrow \infty} x_{2 n+1}=\lim _{n \rightarrow \infty} f\left(x_{2 n}, y_{2 n}\right)=f\left(\lim _{n \rightarrow \infty} x_{2 n}, \lim _{n \rightarrow \infty} y_{2 n}\right)=f(x, y)
$$

and

$$
y=\lim _{n \rightarrow \infty} y_{2 n+1}=\lim _{n \rightarrow \infty} f\left(y_{2 n}, x_{2 n}\right)=f\left(\lim _{n \rightarrow \infty} y_{2 n}, \lim _{n \rightarrow \infty} x_{2 n}\right)=f(y, x) .
$$

Now using (2.1) we have

$$
\begin{aligned}
& S(f(x, y), f(x, y), g(x, y))+S(f(y, x), f(y, x), g(y, x)) \\
& \leq \frac{p}{2} D((x, y),(x, y),(x, y))+\frac{q}{2} D((x, y),(x, y),(f(x, y), f(y, x))) \\
&+\frac{r}{2} D((x, y),(x, y),(g(x, y), g(y, x)))+\frac{s}{2} D((x, y),(x, y),(g(x, y), g(y, x))) \\
&+\frac{s}{2} D((x, y),(x, y),(f(x, y), f(y, x))) \\
&+\frac{p}{2} D((y, x),(y, x),(y, x))+\frac{q}{2} D((y, x),(y, x),(f(y, x), f(x, y))) \\
&+\frac{r}{2} D((y, x),(y, x),(g(y, x), g(x, y)))+\frac{s}{2} D((y, x),(y, x),(g(y, x), g(x, y))) \\
&+\frac{s}{2} D((y, x),(y, x),(f(y, x), f(x, y))) \\
&= \frac{p}{2} D((x, y),(x, y),(x, y))+\frac{q}{2} D((x, y),(x, y),(x, y)) \\
&+\frac{r}{2} D((x, y),(x, y),(g(x, y), g(y, x)))+\frac{s}{2} D((x, y),(x, y),(g(x, y), g(y, x))) \\
&+\frac{r}{2} D((x, y),(x, y),(g(x, y), g(y, x)))+\frac{s}{2} D((x, y),(x, y),(g(x, y), g(y, x))) \\
&+\frac{s}{2} D((x, y),(x, y),(x, y)) \\
&+\frac{p}{2} D((y, x),(y, x),(y, x))+\frac{q}{2} D((y, x),(y, x),(y, x)) \\
&+\frac{r}{2} D((y, x),(y, x),(g(y, x), g(x, y)))+\frac{s}{2} D((y, x),(y, x),(g(y, x), g(x, y))) \\
& \frac{r}{2} D((y, x),(y, x),(g(y, x), g(x, y)))+\frac{s}{2} D((y, x),(y, x),(g(y, x), g(x, y))) . \\
&
\end{aligned}
$$

Therefore,

$$
\begin{aligned}
& S(f(x, y), f(x, y), g(x, y))+S(f(y, x), f(y, x), g(y, x)) \\
& \quad \leq(r+s)(S(x, x, g(x, y))+S(y, y, g(y, x))) .
\end{aligned}
$$

That is,

$$
S(x, x, g(x, y))+S(y, y, g(y, x)) \leq(r+s)(S(x, x, g(x, y))+S(y, y, g(y, x))) .
$$


Since $0 \leq r+s<1$, we get $S(x, x, g(x, y))=S(y, y, g(y, x))=0$. That is, $g(x, y)=x$ and $g(y, x)=y$. Therefore, $(x, y)$ is a coupled common fixed point of $f$ and $g$.

Case 2.2. $g$ is continuous. We can also prove that $(x, y)$ is a coupled common fixed point of $f$ and $g$ similarly as in Case 2.1.

Case 2.3. $X$ satisfies two assumptions (a) and (b). Then by (2.3) we get $x_{n} \preceq x$ and $y \preceq y_{n}$ for all $n \in \mathbb{N}$. By using Lemma 1.4 and Lemma 1.13, we have

$$
\begin{aligned}
D( & (x, y),(x, y),(f(x, y), f(y, x))) \\
\leq & 2 D\left((x, y),(x, y),\left(x_{2 n+2}, y_{2 n+2}\right)\right)+D\left(\left(x_{2 n+2}, y_{2 n+2}\right),\left(x_{2 n+2}, y_{2 n+2}\right),(f(x, y), f(y, x))\right) \\
= & 2 D\left((x, y),(x, y),\left(x_{2 n+2}, y_{2 n+2}\right)\right)+D\left(\left(g\left(x_{2 n+1}, y_{2 n+1}\right), g\left(y_{2 n+1}, x_{2 n+1}\right)\right),\right. \\
& \left.\left(g\left(x_{2 n+1}, y_{2 n+1}\right), g\left(y_{2 n+1}, x_{2 n+1}\right)\right),(f(x, y), f(y, x))\right) \\
\leq & 2 D\left((x, y),(x, y),\left(x_{2 n+2}, y_{2 n+2}\right)\right)+S\left(g\left(x_{2 n+1}, y_{2 n+1}\right), g\left(x_{2 n+1}, y_{2 n+1}\right), f(x, y)\right) \\
& +S\left(g\left(y_{2 n+1}, x_{2 n+1}\right), g\left(y_{2 n+1}, x_{2 n+1}\right), f(y, x)\right) \\
= & 2 S\left(x, x, x_{2 n+2}\right)+2 S\left(y, y, y_{2 n+2}\right)+S\left(g\left(x_{2 n+1}, y_{2 n+1}\right), g\left(x_{2 n+1}, y_{2 n+1}\right), f(x, y)\right) \\
& +S\left(f(y, x), f(y, x), g\left(y_{2 n+1}, x_{2 n+1}\right)\right) .
\end{aligned}
$$

By interchanging the roles of $f$ and $g$ and using (2.1), we have

$$
\begin{aligned}
& S\left(g\left(x_{2 n+1}, y_{2 n+1}\right), g\left(x_{2 n+1}, y_{2 n+1}\right), f(x, y)\right) \\
& \leq \frac{p}{2} D\left(\left(x_{2 n+1}, y_{2 n+1}\right),\left(x_{2 n+1}, y_{2 n+1}\right),(x, y)\right) \\
&+\frac{q}{2} D\left(\left(x_{2 n+1}, y_{2 n+1}\right),\left(x_{2 n+1}, y_{2 n+1}\right),\left(g\left(x_{2 n+1}, y_{2 n+1}\right), g\left(y_{2 n+1}, x_{2 n+1}\right)\right)\right) \\
&+\frac{r}{2} D((x, y),(x, y),(f(x, y), f(y, x))) \\
&+\frac{s}{2} D\left(\left(x_{2 n+1}, y_{2 n+1}\right),\left(x_{2 n+1}, y_{2 n+1}\right),(f(x, y), f(y, x))\right) \\
&+\frac{s}{2} D\left((x, y),(x, y),\left(g\left(x_{2 n+1}, y_{2 n+1}\right), g\left(y_{2 n+1}, x_{2 n+1}\right)\right)\right) \\
&= \frac{p}{2} D\left(\left(x_{2 n+1}, y_{2 n+1}\right),\left(x_{2 n+1}, y_{2 n+1}\right),(x, y)\right) \\
&+\frac{q}{2} D\left(\left(x_{2 n+1}, y_{2 n+1}\right),\left(x_{2 n+1}, y_{2 n+1}\right),\left(x_{2 n+2}, y_{2 n+2}\right)\right) \\
&+\frac{r}{2} D((x, y),(x, y),(f(x, y), f(y, x))) \\
&+\frac{s}{2} D\left(\left(x_{2 n+1}, y_{2 n+1}\right),\left(x_{2 n+1}, y_{2 n+1}\right),(f(x, y), f(y, x))\right) \\
&+\frac{s}{2} D\left((x, y),(x, y),\left(x_{2 n+2}, y_{2 n+2}\right)\right) .
\end{aligned}
$$

Again, by using (2.1), we have

$$
\begin{aligned}
& S\left(f(y, x), f(y, x), g\left(y_{2 n+1}, x_{2 n+1}\right)\right) \\
& \quad \leq \frac{p}{2} D\left((y, x),(y, x),\left(y_{2 n+1}, x_{2 n+1}\right)\right)+\frac{q}{2} D((y, x),(y, x),(f(y, x), f(x, y)))
\end{aligned}
$$




$$
\begin{aligned}
+ & \frac{r}{2} D\left(\left(y_{2 n+1}, x_{2 n+1}\right),\left(y_{2 n+1}, x_{2 n+1}\right),\left(g\left(y_{2 n+1}, x_{2 n+1}\right), g\left(x_{2 n+1}, y_{2 n+1}\right)\right)\right) \\
+ & \frac{s}{2} D\left((y, x),(y, x),\left(g\left(y_{2 n+1}, x_{2 n+1}\right), g\left(x_{2 n+1}, y_{2 n+1}\right)\right)\right) \\
+ & \frac{s}{2} D\left(\left(y_{2 n+1}, x_{2 n+1}\right),\left(y_{2 n+1}, x_{2 n+1}\right),(f(y, x), f(x, y))\right) \\
= & \frac{p}{2} D\left((y, x),(y, x),\left(y_{2 n+1}, x_{2 n+1}\right)\right)+\frac{q}{2} D((y, x),(y, x),(f(y, x), f(x, y))) \\
& +\frac{r}{2} D\left(\left(y_{2 n+1}, x_{2 n+1}\right),\left(y_{2 n+1}, x_{2 n+1}\right),\left(y_{2 n+2}, x_{2 n+2}\right)\right)+\frac{s}{2} D\left((y, x),(y, x),\left(y_{2 n+2}, x_{2 n+2}\right)\right) \\
& +\frac{s}{2} D\left(\left(y_{2 n+1}, x_{2 n+1}\right),\left(y_{2 n+1}, x_{2 n+1}\right),(f(y, x), f(x, y))\right) .
\end{aligned}
$$

It follows from (2.13), (2.14) and (2.15) that

$$
\begin{aligned}
& D((x, y),(x, y),(f(x, y), f(y, x))) \\
& \leq S\left(x, x, x_{2 n+2}\right)+2 S\left(y, y, y_{2 n+2}\right)+\frac{p}{2} D\left(\left(x_{2 n+1}, y_{2 n+1}\right),\left(x_{2 n+1}, y_{2 n+1}\right),(x, y)\right) \\
&+\frac{q}{2} D\left(\left(x_{2 n+1}, y_{2 n+1}\right),\left(x_{2 n+1}, y_{2 n+1}\right),\left(x_{2 n+2}, y_{2 n+2}\right)\right)+\frac{r}{2} D((x, y),(x, y),(f(x, y), f(y, x))) \\
&+\frac{s}{2} D\left(\left(x_{2 n+1}, y_{2 n+1}\right),\left(x_{2 n+1}, y_{2 n+1}\right),(f(x, y), f(y, x))\right)+\frac{s}{2} D\left((x, y),(x, y),\left(x_{2 n+2}, y_{2 n+2}\right)\right) \\
&+\frac{p}{2} D\left((y, x),(y, x),\left(y_{2 n+1}, x_{2 n+1}\right)\right)+\frac{q}{2} D((y, x),(y, x),(f(y, x), f(x, y))) \\
&+\frac{r}{2} D\left(\left(y_{2 n+1}, x_{2 n+1}\right),\left(y_{2 n+1}, x_{2 n+1}\right),\left(y_{2 n+2}, x_{2 n+2}\right)\right)+\frac{s}{2} D\left((y, x),(y, x),\left(y_{2 n+2}, x_{2 n+2}\right)\right) \\
&+\frac{s}{2} D\left(\left(y_{2 n+1}, x_{2 n+1}\right),\left(y_{2 n+1}, x_{2 n+1}\right),(f(y, x), f(x, y))\right) .
\end{aligned}
$$

By using Lemma 1.7 and taking the limit as $n \rightarrow \infty$ in (2.16), we have

$$
\begin{aligned}
& D((x, y),(x, y),(f(x, y), f(y, x))) \\
& \leq 2 S(x, x, x)+2 S(y, y, y)+\frac{p}{2} D((x, y),(x, y),(x, y))+\frac{q}{2} D((x, y),(x, y),(x, y)) \\
&+\frac{r}{2} D((x, y),(x, y),(f(x, y), f(y, x)))+\frac{s}{2} D((x, y),(x, y),(f(x, y), f(y, x))) \\
&+\frac{s}{2} D((x, y),(x, y),(x, y))+\frac{p}{2} D((y, x),(y, x),(y, x)) \\
&+\frac{q}{2} D((y, x),(y, x),(f(y, x), f(x, y)))+\frac{r}{2} D((y, x),(y, x),(y, x)) \\
&+\frac{s}{2} D((y, x),(y, x),(y, x))+\frac{s}{2} D((y, x),(y, x),(f(y, x), f(x, y))) \\
&= \frac{r+s}{2} D((x, y),(x, y),(f(x, y), f(y, x)))+\frac{q+s}{2} D((y, x),(y, x),(f(y, x), f(x, y))) .
\end{aligned}
$$

It implies that

$$
\begin{aligned}
& S(x, x, f(x, y))+S(y, y, f(y, x)) \\
& \quad \leq \frac{r+s}{2}(S(x, x, f(x, y))+(y, y, f(y, x)))+\frac{q+s}{2}(S(y, y, f(y, x))+S(x, x, f(x, y))) \\
& \quad=\frac{r+q+2 s}{2}(S(x, x, f(x, y))+(y, y, f(y, x))) .
\end{aligned}
$$


Since $\frac{r+q+2 s}{2}<1$, we have $S(x, x, f(x, y))+S(y, y, f(y, x))=0$, that is, $f(x, y)=x$ and $f(y, x)=y$. Similarly, one can show that $g(x, y)=x$ and $g(y, x)=y$. This proves that $(x, y)$ is a coupled common fixed point of $f$ and $g$.

From Theorem 2.1, we have following corollaries.

Corollary 2.2 [27, Theorems 2.1 and 2.2] Let $(X, \preceq, d)$ be a partially ordered metric space; $f, g: X \times X \rightarrow X$ be two maps such that

1. $X$ is complete;

2. The pair $(f, g)$ has the mixed weakly monotone property on $X ; x_{0} \preceq f\left(x_{0}, y_{0}\right)$, $f\left(y_{0}, x_{0}\right) \preceq y_{0}$ or $x_{0} \preceq g\left(x_{0}, y_{0}\right), g\left(y_{0}, x_{0}\right) \preceq y_{0}$ for some $x_{0}, y_{0} \in X$;

3. There exist $p, q, r, s \geq 0$ satisfying $p+q+r+2 s<1$ and

$$
\begin{aligned}
& d(f(x, y), g(u, v)) \\
& \leq \frac{p}{2} D_{d}((x, y),(u, v))+\frac{q}{2} D_{d}((x, y),(f(x, y), f(y, x))) \\
& \quad+\frac{r}{2} D_{d}((u, v),(g(u, v), g(v, u)))+\frac{s}{2} D_{d}((x, y),(g(u, v), g(v, u))) \\
& \quad+\frac{s}{2} D_{d}((u, v),(f(x, y), f(y, x)))
\end{aligned}
$$

for all $x, y, u, v \in X$ with $x \preceq u$ and $y \succeq v$, where $D_{d}$ is defined as in Lemma 1.12;

4. $f$ or $g$ is continuous or $X$ has the following property:

(a) If $\left\{x_{n}\right\}$ is an increasing sequence with $x_{n} \rightarrow x$, then $x_{n} \preceq x$ for all $n \in \mathbb{N}$;

(b) If $\left\{x_{n}\right\}$ is an decreasing sequence with $x_{n} \rightarrow x$, then $x \preceq x_{n}$ for all $n \in \mathbb{N}$.

Then $f$ and $g$ have a coupled common fixed point in $X$.

Proof It is a direct consequence of Lemma 1.10, Remark 1.14 and Theorem 2.1.

For similar results of the following for maps on metric spaces and cone metric spaces, the readers may refer to [6, Theorems 2.1, 2.2, 2.4 and 2.6] and [28, Theorem 3.1].

Corollary 2.3 Let $(X, \preceq, S)$ be a partially ordered $S$-metric space and $f: X \times X \rightarrow X$ be a map such that

1. $X$ is complete;

2. $f$ has the mixed monotone property on $X ; x_{0} \preceq f\left(x_{0}, y_{0}\right)$ and $f\left(y_{0}, x_{0}\right) \preceq y_{0}$ for some $x_{0}, y_{0} \in X$

3. There exist $p, q, r, s \geq 0$ satisfying $p+q+r+2 s<1$ and

$$
\begin{aligned}
& S(f(x, y), f(x, y), f(u, v)) \\
& \leq \frac{p}{2} D((x, y),(x, y),(u, v))+\frac{q}{2} D((x, y),(x, y),(f(x, y), f(y, x))) \\
&+\frac{r}{2} D((u, v),(u, v),(f(u, v), f(v, u)))+\frac{s}{2} D((x, y),(x, y),(f(u, v), f(v, u))) \\
&+\frac{s}{2} D((u, v),(u, v),(f(x, y), f(y, x)))
\end{aligned}
$$

for all $x, y, u, v \in X$ with $x \preceq u$ and $y \succeq v$;

4. $f$ is continuous or $X$ has the following property: 
(a) If $\left\{x_{n}\right\}$ is an increasing sequence with $x_{n} \rightarrow x$, then $x_{n} \preceq x$ for all $n \in \mathbb{N}$;

(b) If $\left\{x_{n}\right\}$ is an decreasing sequence with $x_{n} \rightarrow x$, then $x \preceq x_{n}$ for all $n \in \mathbb{N}$.

Then $f$ has a coupled fixed point in $X$.

Proof By choosing $g=f$ in Theorem 2.1 and using Remark 1.20, we get the conclusion.

Corollary 2.4 Let $(X, \preceq, S)$ be a partially ordered S-metric space and $f: X \times X \rightarrow X$ be a map such that

1. $X$ is complete;

2. $f$ has the mixed monotone property on $X ; x_{0} \preceq f\left(x_{0}, y_{0}\right)$ and $f\left(y_{0}, x_{0}\right) \preceq y_{0}$ for some

$x_{0}, y_{0} \in X$

3. There exists $k \in[0,1)$ satisfying

$$
S(f(x, y), f(x, y), f(u, v)) \leq \frac{k}{2}(S(x, x, u)+S(y, y, v))
$$

for all $x, y, u, v \in X$ with $x \preceq u$ and $y \succeq v$;

4. $f$ is continuous or $X$ has the following property:

(a) If $\left\{x_{n}\right\}$ is an increasing sequence with $x_{n} \rightarrow x$, then $x_{n} \preceq x$ for all $n \in \mathbb{N}$;

(b) If $\left\{x_{n}\right\}$ is an decreasing sequence with $x_{n} \rightarrow x$, then $x \preceq x_{n}$ for all $n \in \mathbb{N}$.

Then $f$ has a coupled fixed point in $X$.

Proof By choosing $g=f$ and $p=k, q=r=s=0$ in Theorem 2.1 and using Remark 1.20, we get the conclusion.

Corollary 2.5 Assume that $X$ is a totally ordered set in addition to the hypotheses of Theorem 2.1; in particular, Corollary 2.3, Corollary 2.4. Then $f$ and $g$ have a unique coupled common fixed point $(x, y)$ and $x=y$.

Proof By Theorem 2.1, $f$ and $g$ have a coupled common fixed point $(x, y)$. Let $(z, t)$ be another coupled common fixed point of $f$ and $g$. Without loss of generality, we may assume that $(x, y) \preceq(z, t)$. Then by $(2.1)$ and Lemma 1.3 , we have

$$
\begin{aligned}
& D((x, y),(x, y),(z, t)) \\
&=S(x, x, z)+S(y, y, t) \\
&=S(f(x, y), f(x, y), g(z, t))+S(f(y, x), f(y, x), g(t, z)) \\
& \leq \frac{p}{2} D((x, y),(x, y),(z, t))+\frac{q}{2} D((x, y),(x, y),(f(x, y), f(y, x))) \\
&+\frac{r}{2} D((z, t),(z, t),(g(z, t), g(t, z)))+\frac{s}{2} D((x, y),(x, y),(g(z, t), g(t, z))) \\
&+\frac{s}{2} D((z, t),(z, t),(f(x, y), f(y, x)))+\frac{p}{2} D((y, x),(y, x),(t, z)) \\
&+\frac{q}{2} D((y, x),(y, x),(f(y, x), f(x, y)))+\frac{r}{2} D((t, z),(t, z),(g(t, z), g(z, t))) \\
&+\frac{s}{2} D((y, x),(y, x),(g(t, z), g(z, t)))+\frac{s}{2} D((t, z),(t, z),(f(y, x), f(x, y))) \\
&= \frac{p}{2} D((x, y),(x, y),(z, t))+\frac{q}{2} D((x, y),(x, y),(x, y))+\frac{r}{2} D((z, t),(z, t),(z, t))
\end{aligned}
$$




$$
\begin{aligned}
+ & \frac{s}{2} D((x, y),(x, y),(z, t))+\frac{s}{2} D((z, t),(z, t),(x, y))+\frac{p}{2} D((y, x),(y, x),(t, z)) \\
+ & \frac{q}{2} D((y, x),(y, x),(y, x))+\frac{r}{2} D((t, z),(t, z),(t, z))+\frac{s}{2} D((y, x),(y, x),(t, z)) \\
& +\frac{s}{2} D((t, z),(t, z),(y, x)) \\
= & \frac{p}{2} D((x, y),(x, y),(z, t))+\frac{s}{2} D((x, y),(x, y),(z, t))+\frac{s}{2} D((z, t),(z, t),(x, y)) \\
& +\frac{p}{2} D((y, x),(y, x),(t, z))+\frac{s}{2} D((y, x),(y, x),(t, z))+\frac{s}{2} D((t, z),(t, z),(y, x)) \\
= & \frac{p+2 s}{2}(D((x, y),(x, y),(z, t))+D((y, x),(y, x),(t, z))) \\
= & (p+2 s)(S(x, x, z)+S(y, y, t)) .
\end{aligned}
$$

Since $p+2 s<1$, we have $S(x, x, z)+S(y, y, t)=0$. Then $x=z$ and $y=t$. This proves that the coupled common fixed point of $f$ and $g$ is unique.

Moreover, by using (2.1) and Lemma 1.3 again, we get

$$
\begin{aligned}
S(x, x, y)= & S(f(x, y), f(x, y), g(y, x)) \\
\leq & \frac{p}{2} D((x, y),(x, y),(y, x))+\frac{q}{2} D((x, y),(x, y),(f(x, y), f(y, x))) \\
& +\frac{r}{2} D((y, x),(y, x),(g(y, x), g(x, y)))+\frac{s}{2} D((x, y),(x, y),(g(y, x), g(x, y))) \\
& +\frac{s}{2} D((y, x),(y, x),(f(x, y), f(y, x))) \\
= & \frac{p}{2} D((x, y),(x, y),(y, x))+\frac{s}{2} D((x, y),(x, y),(y, x))+\frac{s}{2} D((y, x),(y, x),(x, y)) \\
= & \frac{p+2 s}{2} D((x, y),(x, y),(y, x)) \\
= & (p+2 s) S(x, x, y) .
\end{aligned}
$$

Since $p+2 s<1$, we get $S(x, x, y)=0$, that is, $x=y$.

Finally, we give an example to demonstrate the validity of the above results.

Example 2.6 Let $X=\mathbb{R}$ with the $S$-metric as in Example 1.6 and the usual order $\leq$. Then $X$ is a totally ordered, complete $S$-metric space. For all $x, y \in X$, put

$$
f(x, y)=g(x, y)=\frac{2 x-y+11}{12}
$$

Then the pair $(f, g)$ has the mixed weakly monotone property and

$$
\begin{aligned}
S(f(x, y), f(x, y), g(u, v)) & =2|f(x, y)-g(u, v)| \\
& =2\left|\frac{2 x-y+11}{12}-\frac{2 u-v+11}{12}\right| \\
& \leq \frac{1}{6}|x-u|+\frac{1}{12}|y-v| \\
& \leq \frac{1}{6}(|x-u|+|y-v|) .
\end{aligned}
$$


Then the contraction (2.1) is satisfied with $p=\frac{1}{6}$ and $q=r=s=0$. Note that other assumptions of Corollary 2.5 are also satisfied and $(1,1)$ is the unique common fixed point of $f$ and $g$.

\section{Competing interests}

The author declares that they have no competing interests.

\section{Acknowledgements}

The author would like to thank the referees for their valuable comments.

Received: 31 July 2012 Accepted: 21 February 2013 Published: 8 March 2013

\section{References}

1. Gähler, S: Über die uniformisierbarkeit 2-metrischer Räume. Math. Nachr. 28, 235-244 (1965)

2. Mustafa, Z, Sims, B: A new approach to generalized metric spaces. J. Nonlinear Convex Anal. 7(2), $289-297$ (2006)

3. Sedghi, S, Shobe, N, Zhou, H: A common fixed point theorem in D*-metric spaces. Fixed Point Theory Appl. 2007, Article ID 27906 (2007)

4. Bukatin, M, Kopperman, R, Matthews, S, Pajoohesh, H: Partial metric spaces. Am. Math. Mon. 116, 708-718 (2009)

5. Huang, LG, Zhang, X: Cone metric spaces and fixed point theorems of contractive mappings. J. Math. Anal. Appl. 332, 1468-1476 (2007)

6. Bhaskar, TG, Lakshmikantham, V: Fixed point theorems in partially ordered metric spaces and applications. Nonlinear Anal. 65(7), 1379-1393 (2006)

7. Sintunavarat, W, Radenović, S, Golubović, Z, Kumam, P: Coupled fixed point theorems for F-invariant set and applications. Appl. Math. Inf. Sci. 7(1), 247-255 (2013)

8. Abbas, M, Nazir, T, Radenović, S: Common coupled fixed points of generalized contractive mappings in partially ordered metric spaces. Positivity (2013). doi:10.1007/s11117-012-0219-z

9. Kadelburg, Z, Nashine, HK, Radenović, S: Coupled fixed point results under tvs-cone metric and $w$-cone-distance. Bull. Math. Anal. Appl. 2(2), 51-63 (2012)

10. Kadelburg, Z, Radenović, S: Common coupled fixed point results in partially ordered Gmetric spaces. Adv. Fixed Point Theory 2, 29-36 (2012)

11. Radenović, S, Pantelić, S, Salimi, P, Vujaković, J: A note on some tripled coincidence point results in G-metric spaces. Int. J. Math. Sci. Eng. App. 6(VI), 23-28 (2012)

12. Rajić, VČ, Radenović, S: A note on tripled fixed point of $w$-compatible mappings in tvs-cone metric spaces. Thai J. Math. (2013, accepted paper)

13. Nashine, HK, Kadelburg, Z, Radenović, S: Coupled common fixed point theorems for $w^{*}$-compatible mappings in ordered cone metric spaces. Appl. Math. Comput. 218, 5422-5432 (2012)

14. Jleli, M, Rajić, VČ, Samet, B, Vetro, C: Fixed point theorems on ordered metric spaces and applications to nonlinear elastic beam equations. J. Fixed Point Theory Appl. (2012). doi:10.1007/s11784-012-0081-4

15. Golubović, Z, Kadelburg, Z, Radenović, S: Coupled coincidence points of mappings in ordered partial metric spaces. Abstr. Appl. Anal. 2012, Article ID 192581 (2012)

16. Dorić, D, Kadelburg, Z, Radenović, S: Coupled fixed point results for mappings without mixed monotone property. Appl. Math. Lett. 25, 1803-1808 (2012)

17. Ding, HS, Li, L, Radenovic, S: Coupled coincidence point theorems for generalized nonlinear contraction in partially ordered metric spaces. Fixed Point Theory Appl. 2012, 96 (2012)

18. Altun, I, Acar, O: Fixed point theorems for weak contractions in the sense of Berinde on partial metric spaces. Topol. Appl. 159, 2642-2648 (2012)

19. Aydi, H, Karapinar, E: A Meir-Keeler common type fixed point theorem on partial metric spaces. Fixed Point Theory Appl. 2012, 26 (2012)

20. Berinde, V: Generalized coupled fixed point theorems for mixed monotone mappings in partially ordered metric spaces. Nonlinear Anal. 74, 18 (2011)

21. Berinde, V, Vetro, F: Common fixed points of mappings satisfying implicit contractive conditions. Fixed Point Theory Appl. 2012, $105(2012)$

22. Choudhurya, BS, Kundu, A: A coupled coincidence point result in partially ordered metric spaces for compatible mappings. Nonlinear Anal. 73, 2524-2531 (2010)

23. Lakshmikantham, V, Ćirić, L: Coupled fixed point theorems for nonlinear contractions in partially ordered metric spaces. Nonlinear Anal. 70(12), 4341-4349 (2009)

24. Sedghi, S, Shobe, N, Aliouche, A: A generalization of fixed point theorem in S-metric spaces. Mat. Vesn. 64(3), 258-266 (2012)

25. An, TV, Dung, NV: Two fixed point theorems in S-metric spaces. Preprint (2012)

26. Sedghi, S, Dung, NV: Fixed point theorems on S-metric spaces. Mat. Vesnik (2012, accepted paper)

27. Gordji, ME, Ramezani, M, Cho, YJ, Akbartabar, E: Coupled common fixed point theorems for mixed weakly monotone mappings in partially ordered metric spaces. Fixed Point Theory Appl. 2012, 95 (2012)

28. Kadelburg, Z, Pavlović, M, Radenović, S: Common fixed point theorems for ordered contractions and quasicontractions in ordered cone metric spaces. Comput. Math. Appl. 59, 3148-3159 (2010) 